\title{
Distribution, characteristics, and prognostic of AIDS and non-AIDS cancers in HIV-infected patients, the ONCOVIH study E Lanoy*1, JP Spano $^{2}$, F Bonnet ${ }^{3,4}$, F Boué ${ }^{2}$, C Katlama ${ }^{1,6}$ and D Costagliola ${ }^{1,6}$
}

\begin{abstract}
Address: ${ }^{1}$ INSERM, U720; UPMC Univ Paris 06, Paris, France, ${ }^{2}$ UPMC Univ Paris 06; AP-HP, Groupe hospitalier Pitié-Salpêtrière, Service de Cancérologie Médicale, Paris, France, ${ }^{3} \mathrm{CHU}$ de Bordeaux, Hôpital Saint-André, Service de Médecine Interne et Maladies Infectieuses, Bordeaux, France, ${ }^{4}$ INSERM, U897; Université Victor Segalen Bordeaux 2, Bordeaux, France, ${ }^{5}$ Université Paris Sud; AP-HP, Hôpital Antoine Béclère, Service de médecine interne, Clamart, France and ${ }^{6} \mathrm{AP}-\mathrm{HP}$, Groupe hospitalier Pitié-Salpêtrière, Service de Maladies Infectieuses et Tropicales, Paris, France * Corresponding author
\end{abstract}

from I I th International Conference on Malignancies in AIDS and Other Acquired Immunodeficiencies (ICMAOI): Basic, Epidemiologic, and Clinical Research

Bethesda, MD, USA. 6-7 October 2008

Published: 17 June 2009

Infectious Agents and Cancer 2009, 4(Suppl 2):OI5 doi:I0.I I86/I750-9378-4-S2-OI5

This abstract is available from: http://www.infectagentscancer.com/content/4/S2/OI5

(c) 2009 Lanoy et al; licensee BioMed Central Ltd.

\section{Background}

With the advent of combined antiretroviral therapy (CART) in 1996 and the decreased incidence of AIDS and death, growing concerns about cancer and in particular non-AIDS-defining cancers have emerged. For instance, in a mortality study conducted in France in 2005, AIDS malignancies including Non-Hodgkin lymphomas (NHL), Kaposi sarcoma and cervical cancer still represented 14 percent of the causes of death among HIVinfected persons and the part of non-AIDS-defining cancers rose from 13 percent in 2000 to 21 percent in 2005. ONCOVIH is a cross-sectional study based on the prospective reporting of new cases of malignant tumors occurring in patients with HIV infection, observed in France, during a 12-month period, year 2006.

\section{Methods}

The main objectives of ONCOVIH were:

- to prospectively record all the new cases of malignancies in HIV-infected patients in 2006 in France;

- to describe the main characteristics of HIV infection (CD4 cell count and nadir, history of antiretroviral therapy, plasma HIV-RNA, AIDS stage), cancer (personal and family history of cancer, viral co-infections, histology and staging of the tumor), and of therapeutic management of the cancer; and
- to evaluate the prognosis of HIV-infected patients with malignancies.

The data were collected from more than 300 different care centers involved in the management of patients with either HIV or malignancies. Duplicate records of the same patient from different centers were reconciled for the HIVand tumor-associated data. Clinical follow-up, antiretroviral therapy and characteristics of HIV infection, therapeutic cancer management including chemotherapy, radiotherapy, or immunotherapy and occurrence of death and cause of death were collected every three months during the first year following the diagnosis. Centralized review of diagnoses and classification of the cause of death were done by a team of clinicians and epidemiologists. Data from HIV patients in care in 2006 in France were extracted from the French Hospital database on HIV (ANRS CO4 FHDH).

\section{Results}

In the ONCOVIH study, 694 tumors were reported in 690 patients by 116 clinical centers for an estimated 83,000 HIV infected patients in care. Of those, the case report forms were completed for 673 tumors in 669 patients. Most common cancers were NHL $(21.5 \%, \mathrm{n}=145)$, Kaposi sarcoma $(15.9 \%, \mathrm{n}=107)$, lung cancer $(9.4 \%, \mathrm{n}=$ $63)$, anal cancer $(8.2 \%, \mathrm{n}=55)$, Hodgkin lymphoma $(7.6 \%, \mathrm{n}=51)$, cutaneous non-melanoma $(5.8 \%, \mathrm{n}=49)$ 
and liver cancers $(5.6 \%, \mathrm{n}=38)$. Cervix cancer was diagnosed in 10 women. For AIDS and non-AIDS-defining cancers, median ages at diagnosis were 44 years $(\mathrm{IQR}=$ $38-51)$ and 49 years (IQR $=42-57)$. Twenty percent and $21 \%$ of diagnosed patients were women. For AIDS-defining cancers, the median CD4 cell count at the time of cancer was $194 / \mathrm{mm}^{3}(\mathrm{IQR}=67-359)$ and the nadir CD4 cell count was $135 / \mathrm{mm}^{3}$ (IQR $\left.=47-263\right)$. For non-AIDSdefining cancers, the corresponding figures were 330/ $\mathrm{mm}^{3}(\mathrm{IQR}=192-503)$ and $147 / \mathrm{mm}^{3}(\mathrm{IQR}=51-258)$. The diagnosis of cancer revealed HIV infection in 28 percent and 5 percent of cases. Twenty-three percent and 62 percent were receiving cART and had plasma HIVRNA $<500$ copies/ml, respectively. In FHDH at the same period, the median age of HIV patients in care was 43 years (IQR = 37-49) with 33\% women. The median CD4 cell count was 454 (IQR $=312-634)$, and 67 percent received cART with plasma $H I V-R N A<500$ copies/ml. In the general population, the mean age at diagnosis of cancer was 66 years of age in 2005.

The overall 1-year survival rate was $72 \%(n=185,95 \% \mathrm{CI}$ : $68-75 \%$ ). Prognosis after a diagnosis of lung cancer (1year survival rate: 35\%) hepatocarcinoma (47\%) and NHL $(65 \%)$ were the worst. Most common causes of death were NHL (45), lung cancer (35) and liver diseases (hepatocarcinoma or cirrhosis or hepatic failure, $n=18$ ).

\section{Conclusion}

NHL and Kaposi sarcoma remain the most frequent cancers diagnosed in 2006 in France in HIV-infected people despite the widespread use of CART. However, non-AIDSdefining cancers including lung cancer, anal cancer and Hodgkin lymphoma represent $62 \%$ of diagnosed cancers. Diagnosis of cancer occurred at much younger age than in the general population, in patients with a lower CD4 cell count and more often with detectable plasma HIV RNA than in HIV-infected patients not diagnosed with cancer, suggesting that a better control of HIV and its induced immunodeficiency is required to prevent AIDS and nonAIDS cancer in HIV infected patients in addition to the control of other usual risk factors for cancer.

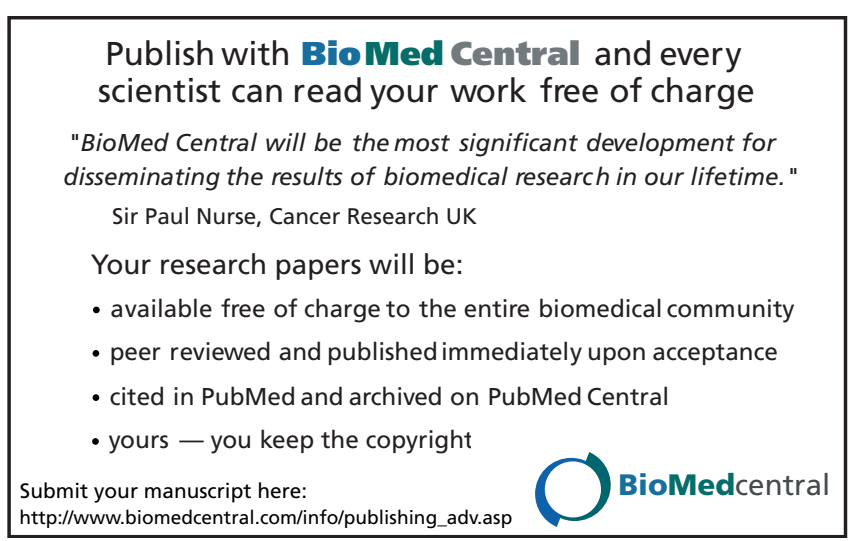

Page 2 of 2

(page number not for citation purposes) 